\title{
Die politische Neutralität der Schule
}

\section{Lorenz Engi *}

Die Rechtsordnungen vieler Kantone sehen vor, dass die öffentliche Schule politisch neutral sei. Die genaue Tragweite dieses Gebots ist wenig klar. Der Beitrag unterbreitet einen Vorschlag zur politischen Neutralität von Lehrpersonen. Demnach dürfen diese unter bestimmten Bedingungen politische Meinungen äussern. Strengere Massstäbe sind bei Lehrmitteln anzulegen.

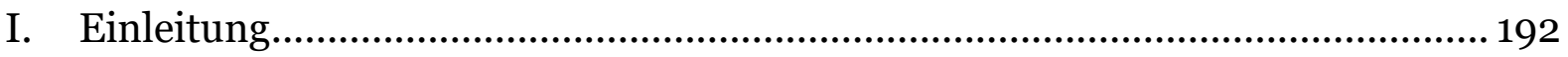

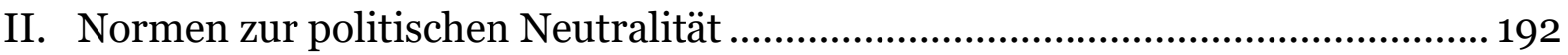

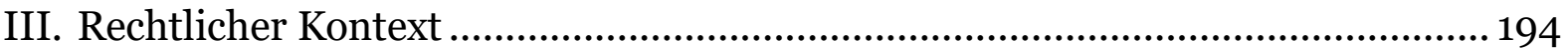

1. Anstellungsvoraussetzungen und Treuepflicht ....................................... 194

2. Grundrechte von Lehrpersonen sowie Schülerinnen und Schülern .............. 195

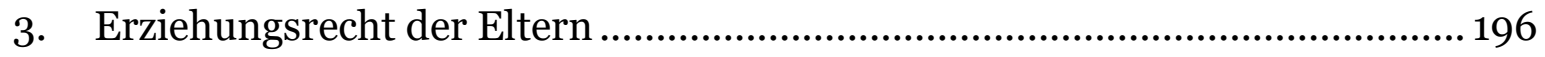

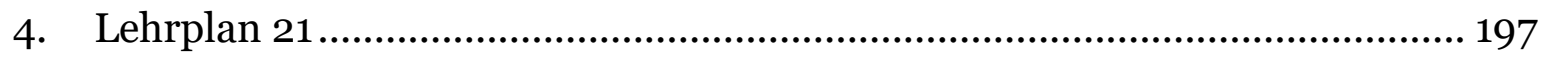

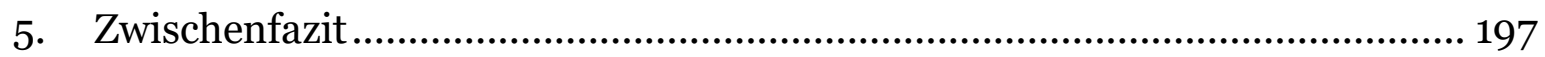

IV. Neutralität des Unterrichts.................................................................... 198

1. Stellungnahmen in der Literatur ......................................................... 198

2. Eigener Vorschlag: Kriterien................................................................ 199

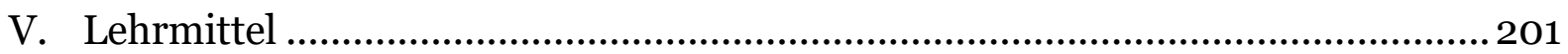

VI. Grundsätzlich: Neutralität als Grenze .....................................................203

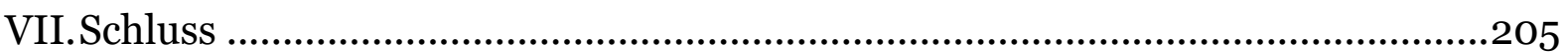

Zitiervorschlag: Lorenz Engi, Die politische Neutralität der Schule, in: sui-generis 2019, S. 191

URL: $\quad$ sui-generis.ch/102

DOI: $\quad$ https://doi.org/10.21257/sg.102

* Lorenz Engi ist Privatdozent an der Universität St. Gallen sowie Dozent an der Zürcher Hochschule für Angewandte Wissenschaften (lorenz.engi@unisg.ch). 


\section{Einleitung}

1 Ist heute von der Neutralität des Staates und insbesondere von der Neutralität der Schule die Rede, so geht es meistens um die religiöse Neutralität. Viel weniger Beachtung findet ein anderer Aspekt: die politische Neutralität der Schule. Zahlreiche kantonale Verfassungen und Gesetze schreiben explizit vor, dass die Schule politisch neutral sei. Diese Normen werfen hinsichtlich der praktischen Umsetzung nicht weniger Fragen auf als die Gebote der religiösen Neutralität - ja in gewisser Weise sind die Probleme bei der politischen Neutralität sogar noch grösser.

Denn während in der Gegenwartsgesellschaft in religiösen Fragen vielfach eine Haltung der Indifferenz vorherrscht, die der religiösen Neutralitätsforderung entgegenkommt, haben so gut wie alle Personen eine politische Grundhaltung und eine Meinung zu konkreten politischen Fragen. Dies ist auch bei Lehrpersonen der Fall, die sich fragen müssen, wie sie in ihrer Unterrichtstätigkeit mit diesen legitimen und natürlichen, ja staatspolitisch erwünschten - Präferenzen umgehen. Während zahlreiche Fächer diesbezüglich wenige Probleme aufwerfen (zum Beispiel naturwissenschaftliche Fächer), gibt es in anderen Fachbereichen zahlreiche Berührungspunkte zu persönlichen Überzeugungen. ${ }^{1}$

3 Die Frage nach der politischen Neutralität der Schule hat darüber hinaus eine grundsätzliche Dimension. Sie berührt die Thematik, ob und inwiefern sich staatliche Angestellte in ihrer beruflichen

Dies betrifft namentlich den Fachbereich «Natur, Mensch, Gesellschaft» (NMG) gemäss Lehrplan 21.
Tätigkeit von ihren persönlichen Auffassungen distanzieren können und müssen. Diese allgemeine Fragestellung kann im vorliegenden Rahmen nicht eigens behandelt werden, soll aber immerhin präsent sein und punktuell berücksichtigt werden.

4 Im Folgenden sollen zunächst die bestehenden Regelungen zur politischen Neutralität der Schule und der rechtliche Kontext, in welchem dieses Gebot steht, beleuchtet werden (nachfolgend Ziff. II und III). Sodann soll die Neutralitätspflicht näher betrachtet und ausgedeutet werden, vor allem in zweierlei Hinsicht: bezüglich der Unterrichtstätigkeit (IV) und der Lehrmittel (V). Soweit dabei auf konkrete rechtliche Gegebenheiten einzugehen ist, konzentrieren sich die folgenden Ausführungen auf die Situation im Kanton Zürich. Nach einer allgemeinen Betrachtung zum Inhalt und zur Reichweite des Neutralitätsgebots (VI) soll ein Fazit gezogen werden (VII).

\section{Normen zur politischen Neutralität}

5 Gemäss Art. 62 Abs. 1 BV sind die Kantone für das Schulwesen zuständig. ${ }^{2}$ Zahlreiche kantonale Erlasse enthalten die Vorschrift, dass die Schule politisch neutral sei. Diese Normen beziehen sich auf die Volksschule sowie Berufs- und

2 Das «Schulwesen» umfasst die gesamte Grundund Mittelschule; Giovanni Biaggini, BV Kommentar - Bundesverfassung der Schweizerischen Eidgenossenschaft, 2. Aufl., Zürich 2017, Art. 62 Rz. 5; Peter Hänni, Kommentar zu Art. 62 BV, in: Bernhard Waldmann/Eva Maria Belser/Astrid Epiney (Hrsg.), Bundesverfassung - Basler Kommentar, Basel 2015, Rz. 13; auch die Hochschulstufe einschliessend Bernhard Ehrenzeller, Kommentar zu Art. 62 BV, in: Bernhard Ehrenzeller u.a. (Hrsg.), Die schweizerische Bundesverfassung - St. Galler Kommentar, 3. Aufl., St. Gallen/Zürich 2015, Rz. 7. 
Mittelschulen (nicht aber die Hochschulen). 3 Zuweilen sind die Vorschriften zur politischen Neutralität auf Verfassungsstufe, teilweise sind sie in Gesetzen verankert. Entsprechende Vorschriften enthalten die Rechtsordnungen der Kantone Aargau4, Basel-Stadt5, Bern 6 , Freiburg7, Genf ${ }^{8}$, Graubünden9, Luzern ${ }^{10}$, Nidwalden $^{11}$, Obwalden ${ }^{12}$, Waadt ${ }^{13}$ und Zü$\operatorname{rich}^{14} .{ }^{15}$

3 So z.B. für den Kanton Zürich Markus Rüssli, Kommentar zu Art. $116 \mathrm{KV} \mathrm{ZH}$, in: Isabelle Häner/Markus Rüssli/Evi Schwarzenbach (Hrsg.), Kommentar zur Zürcher Kantonsverfassung, Zürich/Basel/Genf 2007, Rz. 2.

4 § 2 Abs. 2 Schulgesetz vom 17. März 1981 (SAR 401.100): «Die öffentlichen Schulen sind unselbständige öffentlich-rechtliche Anstalten; sie sind politisch und konfessionell neutral.»

$5 \S 18$ Abs. 2 der Verfassung des Kantons BaselStadt vom 23. März 2005 (SR 131.222.1): «Staatliche Kindergärten, Schulen, Tagesbetreuungseinrichtungen, Sonderschulen und Heime werden konfessionell und politisch neutral geführt.»

6 Art. 43 Abs. 1 der Verfassung des Kantons Bern vom 6. Juni 1993 (SR 131.212): «Kanton und Gemeinden führen öffentliche Kindergärten und Schulen. Der Unterricht ist konfessionell und politisch neutral.»

7 Verfassung des Kantons Freiburg vom 16. Mai 2004 (SR 131.219), Art. 64 Abs. 4 Satz 1 (betr. Grundschulunterricht): «Der Unterricht achtet die konfessionelle und politische Neutralität.»; sowie Art. 2 Abs. 3 des Gesetzes über die obligatorische Schule vom 9. September 2014 (SchG; SGF 411.0.1): «Die Schule achtet die konfessionelle und politische Neutralität.»

8 Art. 11 Abs. 1 und 2 Loi sur l'instruction publique du 17 septembre 2015 (LIP ; $C$ C 11 10): «1 L'enseignement public garantit le respect des convictions politiques et religieuses des élèves et des parents. ${ }^{2}$ A cet égard, toute forme de propagande politique et religieuse est interdite auprès des élèves.»

9 Art. 89 Abs. 1 der Verfassung des Kantons Graubünden vom 18. Mai 2003/14. September 2003 (SR 131.226): "Der Unterricht an den öffentlichen Schulen beruht auf einer christlichhumanistischen Grundlage. Er ist konfessionell und politisch neutral und von Toleranz geprägt.»

10 Gesetz über die Volksschulbildung vom 22. März 1999 (VBG; SRL 400a), §3: «Die öffentliche Volksschule ist politisch und konfessionell neutral.»

11 Art. 2 Abs. 1 Gesetz über das Bildungswesen vom 17. April 2002 (BiG; NG 311.1): «Die öffentlichen
6 Die politische Neutralität ist nicht mit einer absoluten Wertneutralität gleichzusetzen. In den Schul- und Bildungsgesetzen finden sich häufig Bekenntnisse zu christlichen, demokratischen und ähnlichen Wertgrundlagen. So besagt etwa $\S 2$ Abs. 1 Satz 1 des Volksschulgesetzes des Kantons Zürich16: «Die Volksschule erzieht zu einem Verhalten, das sich an christlichen, humanistischen und demokratischen Wertvorstellungen orientiert.»17 Gemäss $\S 2$ Abs. 4 des gleichen Erlasses fördert die Volksschule unter anderem die Achtung vor Mitmenschen und Umwelt. Die Bildungsdirektion des Kantons

Schulen sind politisch neutral. Sie wahren die Glaubens- und Gewissensfreiheit und nehmen auf Minderheiten Rücksicht. Sie fördern Schülerinnen und Schüler gleichermassen.»

12 Art. 10 Abs. 1 Bildungsgesetz vom 16. März 2006 (GDB 410.1): «Die öffentlichen Schulen sind politisch neutral. Sie wahren die Glaubens- und Gewissensfreiheit, nehmen auf Minderheiten Rücksicht und fördern alle Schülerinnen und Schüler sowie Studierenden gleichermassen.»

13 Art. 45 Abs. 2 Verfassung des Kantons Waadt vom 14. April 2003 (SR 131.231): «Dieser Unterricht [der öffentliche Unterricht] ist politisch und konfessionell neutral.»; sowie Art. 9 Abs. 1 Loi sur l'enseignement obligatoire du 7 juin 2011 (LEO; RSV 400.02): «L'enseignement est neutre du point de vue religieux et politique.»

14 Art. 116 Abs. 2 der Verfassung des Kantons Zürich vom 27. Februar 2005 (SR 131.211): «Diese [die öffentlichen Schulen] sind den Grundwerten des demokratischen Staatswesens verpflichtet. Sie sind konfessionell und politisch neutral.» Sowie $\S 4$ Bildungsgesetz vom 1. Juli 2002 (BiG; LS 410.1): «Die staatlichen Schulen sind politisch und konfessionell neutral.»

15 Im Kanton Tessin gibt es bezüglich des Staatskundeunterrichts die Vorschrift: «Il principio della neutralità dell'insegnamento deve essere garantito.» (Art. 23a Abs. 4 Legge della scuola del 1 febbraio 1990 [RL 400.100]).

16 Volksschulgesetz vom 7. Februar 2005 (VSG; LS 412.100).

17 Vgl. z.B. auch Art. 2 Abs. 2 des Volksschulgesetzes vom 19. März 1992 des Kantons Bern (BSG; 432.210): «Sie [die Volkschule] trägt, ausgehend von der christlich-abendländischen und demokratischen Überlieferung, zur harmonischen Entwicklung der Fähigkeiten der jungen Menschen bei.» 
Zürich erläutert diese Bestimmungen folgendermassen: «Die Volksschule ist nicht wertfrei. Ihr gesetzlicher Bildungsund Erziehungsauftrag richtet sich nach bestimmten Werten aus. Die Kantonsverfassung sagt dazu, dass die öffentlichen Schulen den Grundwerten des demokratischen Staatswesens verpflichtet sind (Art. 116 Abs. 2 KV). Diese Werteorientierung, die gesellschaftlich als allgemein anerkannt betrachtet werden kann, entspricht dem Gebot der politischen und konfessionellen Neutralität der Volksschule (Art. 116 Abs. 2 KV; § 4 Bildungsgesetz, BiG).»18

7 Die Normen zur politischen Neutralität, die später genauer zu interpretieren sind, stehen im Zusammenhang mit zahlreichen anderen Vorschriften, die den schulischen Unterricht und die Stellung der Lehrperson bestimmen. Sie sind in diesem Kontext zu sehen, weshalb im Folgenden dieses rechtliche Umfeld näher beleuchtet werden soll.

\section{Rechtlicher Kontext}

\section{Anstellungsvoraussetzungen und Treuepflicht}

8 Das Lehrpersonal staatlicher Schulen untersteht den Normen des öffentlichen Personalrechts. ${ }^{19}$ Grundlage der Tätigkeit

18 Bildungsdirektion des Kantons Zürich - Volksschulamt, Umsetzung Volksschulgesetz. Erläuterungen zum neuen Volksschulgesetz und zur neuen Volksschulverordnung, Zürich 2008, S. 7. Auch im Zürcher Verfassungsrat wurde vielfach betont, dass die politische Neutralität der Schule keine Wertneutralität bedeute; vgl. Protokoll des Zürcher Verfassungsrates, 27. Sitzung, 5. Dezember 2002, S. 1552 ff.

19 Die an der Volksschule tätigen Personen unterstehen im Kanton Zürich dem Lehrpersonalgesetz vom 10. Mai 1999 (LPG; SR 412.31). Dieses verweist, soweit es keine besonderen Bestimmungen bildet ein öffentlich-rechtliches Arbeitsverhältnis zwischen dem Staat (Arbeitgeber) und einer natürlichen Person (Arbeitnehmerin oder Arbeitnehmer). Bei der Begründung eines solchen Arbeitsverhältnisses gelten besondere Regeln. Neben der fachlichen ist auch die persönliche Eignung Voraussetzung für die Anstellung einer Bewerberin oder eines Bewerbers. ${ }^{20}$ Zur persönlichen Eignung gehört das Mittragen von fundamentalen staatlichen Grundsätzen. An dieser Voraussetzung fehlt es einer Lehrperson, wenn die Bewerberin oder der Bewerber durch ihre bzw. seine politische Tätigkeit offensichtlich den freiheitlich-demokratischen Grundwerten des Staates widerspricht und darauf abzielt, diese Werte in rechtswidriger Weise zu untergraben. ${ }^{21}$ Entscheidend ist die Bereitschaft und Fähigkeit, auf die Propagierung politischer und weltanschaulicher Einstellungen gegenüber den Schülerinnen und Schülern zu verzichten und Gewähr zu bieten für neutralen Unterricht. ${ }^{22}$

9 Während der Tätigkeit als staatliche Angestellte bzw. staatlicher Angestellter besteht sodann eine sog. Treuepflicht. Die Tätigkeit verpflichtet zu einem gegenüber dem Gemeinwesen loyalen Verhalten, das die Interessen des Staates nicht beeinträchtigt. ${ }^{23}$ Die Treuepflicht verlangt nicht eine bestimmte Gesin-

enthält, auf das Personalgesetz vom 27. September 1998 (PG; LS 177.10) (§ 2 LPG).

20 § 11 Abs. 1 PG.

${ }^{21}$ Fallsammlung bei Peter Hänni, Das öffentliche Dienstrecht der Schweiz. Dargestellt anhand der Gerichts- und Verwaltungspraxis in Bund und Kantonen, 2. Aufl., Zürich/Basel/Genf 2008, S. 3 ff.

22 Tobias Jaag, Das öffentlichrechtliche Dienstverhältnis im Bund und im Kanton Zürich - ausgewählte Fragen, in: Schweizerisches Zentralblatt für Staats- und Verwaltungsrecht 95 (1994), S. $433-473,445 \mathrm{f}$.

23 Vgl. § 49 PG. 
nung, sondern betrifft nur das äussere Verhalten einer Person. ${ }^{24}$ Sie setzt eine grundsätzlich positive Einstellung zum Staat voraus, bedeutet aber nicht, dass sich Angestellte jeglicher Kritik zu enthalten hätten. ${ }^{25}$ Wird Kritik am staatlichen Handeln geübt, haben sich staatliche Angestellte aber eine gewisse Zurückhaltung aufzuerlegen. ${ }^{26}$

Das Gemeinwesen braucht es nicht hinzunehmen, dass namentlich Lehrkräfte innerhalb oder ausserhalb der Schule Auffassungen vertreten, die den Grundwerten, die sich in der Rechtsordnung und vor allem in der Verfassung manifestieren, zuwiderlaufen. Fundamentale Widersprüche zur staatlichen Grundordnung lägen etwa vor, wenn Lehrpersonen die Abschaffung der wichtigsten Grundrechte oder die Beseitigung der Demokratie postulieren würden. ${ }^{27}$ So wurde es als eine Verletzung der Treuepflicht erachtet, als ein Lehrer in einem Zeitungs-

$24 \overline{\text { Urs Bürgi/Gudrun Bürgi-Schneider, Öffentliches }}$ Personalrecht des Kantons Zürich, in: dies. (Hrsg.), Handbuch öffentliches Personalrecht, Zürich/Basel/Genf 2017, S. 525-608, 545; Yvo Hangartner, Treuepflicht und Vertrauenswürdigkeit der Beamten, in: Schweizerisches Zentralblatt für Staats- und Verwaltungsrecht 85 (1984), S. 385-403, 392; Peter Hänni, Organisationsrecht, Teil 2: Personalrecht des Bundes, 2. Aufl., Basel 2004, Rz. 207; ders., Die Treuepflicht im öffentlichen Dienstrecht, Diss. Freiburg, Freiburg 1982, S. 38; Jaag (Fn. 22), S. 455; Beatrix Schibli, Einschränkungen der Meinungsfreiheit des Bundespersonals, Diss. Zürich 2005, S. $86 \mathrm{f}$.

25 Ulrich Häfelin/Georg Müller/Felix Uhlmann, Allgemeines Verwaltungsrecht, 7. Aufl., Zürich/ St. Gallen 2016, S. 447.

26 Jörg Paul Müller/Markus Schefer, Grundrechte in der Schweiz - Im Rahmen der Bundesverfassung, der EMRK und der UNO-Pakte, 4. Aufl., Bern 2008, S. 405; BGE 136 I 332 E. 3.2.1 S. 336.

27 Herbert Plotke, Schweizerisches Schulrecht, 2. Aufl., Bern 2003, S. 574; Paul Richli, Grundrechtliche Aspekte der Tätigkeit von Lehrkräften, in: Aktuelle Juristische Praxis 6 (1993), S. 673-687, 678. artikel die Steinigung von Ehebrecherinnen auf islamischer Grundlage rechtfertigte. ${ }^{28}$ Dagegen hat das Bundesgericht im Fall eines Dozenten der Zürcher Hochschule der Künste (ZHdK) entschieden, dass dieser die Treuepflicht nicht verletzt habe. Der Dozent hatte sich mit einem Flugblatt gegen das Projekt des Campus der ZHdK auf dem ToniAreal gewehrt, das die Hochschulleitung unterstützte. Er verteilte das Flugblatt vor einer Sitzung des Zürcher Kantonsrates, an der das Thema behandelt wurde. Das Bundesgericht befand, dass sich die Aktion in erster Linie an die Mitglieder des Kantonsrates gewendet habe und nicht primär den Zweck gehabt habe, auf eine Pflichtverletzung der Hochschulleitung hinzuweisen. Die Äusserung sei im Rahmen einer demokratischen Auseinandersetzung erfolgt, und angesichts der Bedeutung der Meinungsäusserungsfreiheit im demokratischen Willensbildungsprozess sei sie zulässig gewesen. ${ }^{29}$

\section{Grundrechte von Lehrpersonen sowie Schülerinnen und Schülern}

11 Im Zusammenhang mit der politischen Neutralität der Schule sind des Weiteren die Grundrechte der Lehrpersonen sowie diejenigen der Schülerinnen und Schüler von Belang. Lehrerinnen und Lehrer können sich grundsätzlich auf die gleichen Grundrechte berufen wie andere natürliche Personen. Im Hinblick auf die politische Neutralität der Schule sind insbesondere die persönliche Freiheit, die Meinungsfreiheit, die Vereinsfreiheit sowie die politischen Rechte von Bedeutung. Aufgrund des Sonderstatusverhält-

$28 \overline{\mathrm{ZBl} \text { 2004, S. } 663 \text { ff. E. 11, S. 665-667. Weiteres }}$ Fallrecht bei Hänni, Das öffentliche Dienstrecht (Fn. 21), S. $111 \mathrm{ff}$.

29 BGE 136 I 332, insb. E. 3.3.5-3.3.7, S. 339 ff. 
nisses oder besonderen Rechtsverhältnisses, in dem sich staatliche Angestellte befinden, ergeben sich jedoch bestimmte Besonderheiten. ${ }^{30}$

Staatliche Angestellte müssen bei der Ausübung ihrer Freiheitsrechte Einschränkungen hinnehmen, die sich aus ihrer Treuepflicht und allenfalls ihren Amtspflichten ergeben. Die Voraussetzungen für die Einschränkungen von Freiheitsrechten gemäss Art. 36 BV müssen dabei erfüllt sein, doch sind die Anforderungen an die gesetzliche Grundlage bezüglich Normstufe und Normdichte und an die Verhältnismässigkeit geringer. ${ }^{31}$ Insbesondere die Meinungsfreiheit kann durch die Treuepflicht eingeschränkt sein. ${ }^{2}$ Einschränkungen derselben sind jedoch nur zulässig, wenn sie sachlich begründet sind und in einem vernünftigen Verhältnis zu deren Zweck stehen. 33

Die Schülerinnen und Schüler sind nicht an das Gebot der politischen Neutralität der Schule gebunden. Ebenso obliegt ihnen keine Treuepflicht. Ihre Meinungsfreiheit kann besonderen Schranken unterworfen werden, soweit Zweck und Betrieb der Schule es erfordern. 34

14 Aus der Meinungsfreiheit ergibt sich nicht nur ein Recht der Schülerinnen und Schüler, im Rahmen der öffentlichen Schule ihre Meinung frei zu äussern. Aus dem Grundrecht fliesst auch der Anspruch, in der Schule nicht in der eigenen

30 Eingehend Markus Müller, Das besondere Rechtsverhältnis, Habil., Bern 2003, insb. S. 24 ff.

31 Häfelin/Müller/Uhlmann (Fn. 25), S. 448.

32 BGE 136 I 332 E. 3.2 S. 335.

33 BGE 136 I 332 E. 3.2.1 S. 335.

34 BGer, Urteil vom 24. Mai 1978, ZBl 1978, S. 505, E. $3 \mathrm{~b} / 5 \mathrm{~b}, \mathrm{~S} .510,513$.
Meinungsbildung unzulässig beeinflusst zu werden. Die Meinungsfreiheit verbietet es dem Staat, den Einzelnen Ansichten durch Indoktrination, einseitige Information oder andere Mittel aufzudrängen.35 Daher wird namentlich von Lehrkräften an öffentlichen Schulen verlangt, dass sie bei politischen, religiösen oder weltanschaulichen Stellungnahmen im Unterricht insoweit Zurückhaltung üben, als die Schülerinnen und Schüler noch nicht in der Lage sind, ihr eigenes Urteil den Wertungen des Lehrers entgegenzuhalten. ${ }^{36}$

\section{Erziehungsrecht der Eltern}

15 Die politische Neutralität der Schule hat ferner einen Bezug zum elterlichen Erziehungsrecht. Gemäss Art. 301 Abs. 1 ZGB37 leiten die Eltern im Blick auf das Wohl des Kindes dessen Pflege und Erziehung. Art. 302 Abs. 1 ZGB bestimmt: «Die Eltern haben das Kind ihren Verhältnissen entsprechend zu erziehen und seine körperliche, geistige und sittliche Entfaltung $\mathrm{zu}$ fördern und $\mathrm{zu}$ schützen.» Gemäss Abs. 3 des gleichen Artikels sollen sie zu diesem Zweck in geeigneter Weise mit der Schule und, wo es die Umstände erfordern, mit der öffentlichen und gemeinnützigen Jugendhilfe zusammenarbeiten.

35 Andreas Kley/Esther Tophinke, Kommentar zu Art. $16 \mathrm{BV}$, in: Bernhard Ehrenzeller/Benjamin Schindler/Rainer J. Schweizer/Klaus A. Vallender (Hrsg.), St. Galler Kommentar zur Schweizerischen Bundesverfassung, 3. Aufl., Zürich/St. Gallen 2014, Rz. 10.

36 Müller/Schefer (Fn. 26), S. 408; Regina Kiener, Bildung, Forschung und Kultur (§ 57), in: Daniel Thürer/Jean-François Aubert/Jörg Paul Müller (Hrsg.), Verfassungsrecht der Schweiz, Zürich 2001, Rz. 3; Heribert Rausch, Die Meinungsäusserungsfreiheit der Staatsangestellten, ZBl 80 (1979), S. 97-115, 112.

37 Schweizerisches Zivilgesetzbuch vom 10. Dezember 1907 (SR 210). 
Der Auftrag der Schule beschränkt sich auf die Ergänzung und Unterstützung der elterlichen Erziehungsbemühungen. ${ }^{8}$ Die Schule hat sich daher zurückzuhalten, wo religiös, weltanschaulich oder parteipolitisch geprägte Werte die Erziehung beeinflussen. 39 Soweit es mit einem geordneten Schulbetrieb vereinbar ist, muss die staatliche Schule auf besonders wichtige Erziehungsanliegen und Wertvorstellungen der Eltern Rücksicht nehmen und zum Beispiel Dispensationen aus religiösen oder anderen Gründen gewähren. ${ }^{40}$ Die Bestrebungen der Schule brauchen aber nicht vollumfänglich mit denen der einzelnen Eltern zu harmonieren.41 Der Erziehungsauftrag der Schule bestimmt sich nach den Interessen der Allgemeinheit und ist auf allgemein geteilte Werte ausgerichtet.42 Es findet notwendig eine gewisse Ausbalancierung mit den Ansichten der Eltern

$38 \overline{\text { Kurt Affolter-Fringeli/Urs Vogel, Schweizerisches }}$ Zivilgesetzbuch - Berner Kommentar, Art. 296327c ZGB, Bern 2016, Art. 302 N. 55; Linus Cantieni/Rolf Vetterli, in: Andrea Büchler/ Dominique Jakob (Hrsg.), Kurzkommentar zum Schweizerischen Zivilgesetzbuch, 2. Aufl., Basel 2018, Art. 302 N. 6; Plotke (Fn. 27), S. 10. In diesem Sinn bestimmt etwa § 35 Abs. 1 der Verfassung des Kantons Aargau vom 25. Juni 1980 (SR 131.227): «Der Unterricht an öffentlichen Schulen hat das Recht der Eltern auf Erziehung und Bildung ihrer Kinder und die Persönlichkeit der Schüler zu achten.»; oder Art. 2 Abs. 1 Satz 1 des Schulgesetzes vom 25. April 2004 des Kantons Appenzell-Innerrhoden (SchG; GS 411.00o): «Die Schulen unterstützen die Inhaber der elterlichen Sorge in der Erziehung des Kindes zu einem selbstständigen, lebensbejahenden und gemeinschaftsfähigen Menschen».

39 Plotke (Fn. 27), S. 31.

40 Judith Wyttenbach, Grund- und Menschenrechtskonflikte zwischen Eltern, Kind und Staat Schutzpflichten des Staates gegenüber Kindern und Jugendlichen aus dem internationalen Menschenrechtsschutz und der Bundesverfassung (Art. 11 BV), Diss. Bern, Basel 2006, S. 261.

41 Plotke (Fn. 27), S. 572.

42 Vgl. bspw. § 2 Abs. 1 des Zürcher Volksschulgesetzes, (Fn. 16). statt. Die Schule erfüllt gerade dadurch eine wichtige Sozialisierungsfunktion, dass Kinder in dieser Institution mit anderen Anschauungen als denen konfrontiert werden, die sie im elterlichen und familiären Umfeld kennenlernen. Die Eltern haben sich daher sowohl der Organisation, als auch dem Lehrplan der öffentlichen Schule weitgehend $\mathrm{zu}$ fügen.43 Eine systematische politische Indoktrination wäre jedoch mit den Rechten der Eltern nicht zu vereinbaren und durch den Erziehungsauftrag der Schule nicht gedeckt.

\section{Lehrplan 21}

17 Von Bedeutung sind im vorliegenden Zusammenhang auch die Vorgaben des Lehrplans 21, besonders im Fachbereich «Natur, Mensch, Gesellschaft».44 Der Lehrplan der Volksschule im Kanton Zürich enthält didaktische Hinweise und behandelt in diesem Zusammenhang auch das Verhalten der Lehrperson. Bezüglich des Fachs „Religionen, Kulturen, Ethik» schreibt er unter anderem vor: «Die Lehrperson ist sich ihres eigenen weltanschaulichen Horizontes bewusst und leitet die Schülerinnen und Schüler transparent mit einer interessierten und respektvollen Haltung zu Erkundungen und Begegnungen an.»45 Die Lehrinhalte sollten unter dem Aspekt der «Mehrperspektivität» behandelt werden. 46

\section{Zwischenfazit}

18 Unabhängig von speziellen Verpflichtungen zur politischen Neutralität bestehen - wie sich gezeigt hat - zahlreiche Nor-

\footnotetext{
43 Wyttenbach (Fn. 40), S. 261.

44 Vgl. www.lernplan21.ch.

45 zh.lehrplan.ch/ $\rightarrow$ Religionen, Kulturen, Ethik $\rightarrow$ Didaktische Hinweise.

46 Ebd.
} 
men, die einer politischen Ausrichtung des schulischen Unterrichts und der Lehrtätigkeit Grenzen setzen. Von Bedeutung ist unter anderem die Treuepflicht der Lehrpersonen, die es verbietet, dass diese sich aktiv gegen fundamentale Normen des Staates wenden. Eigentlich extremistische Strömungen sind aufgrund dieser Normen an öffentlichen Schulen nicht erlaubt, wobei es nicht auf die Gesinnung der jeweiligen Person, sondern auf deren Verhalten ankommt. Eine weitere Grenze ergibt sich aus der Meinungsfreiheit der Schülerinnen und Schüler. Diese vermittelt ein Abwehrrecht gegen eine bewusste Lenkung der Meinungsbildung durch den Staat und staatliche Akteure. Eine eigentliche Indoktrination ist daher unzulässig.

Die Grenzen, die damit gesteckt werden, lassen jedoch weite Spielräume. Die Treuepflicht, die Grundrechte der Schülerinnen und Schüler, das elterliche Erziehungsrecht und weitere Faktoren wie der Lehrplan verhindern nur sehr weitgehende Formen politischer Beeinflussung. Es sind viele Formen politischer Äusserungen von Lehrpersonen denkbar, die mit diesen Normen nicht kollidieren. Das Gebot der politischen Neutralität verspricht, diesen weiten Spielraum etwas einzugrenzen und klarere Handlungsanweisungen zu geben. Im Folgenden soll daher diese rechtliche Forderung näher beleuchtet werden, im Hinblick auf zwei Hauptfelder schulischer Praxis: die Unterrichtstätigkeit und die Lehrmittel.

\section{Neutralität des Unterrichts}

\section{Stellungnahmen in der Literatur}

2o Die Lehrperson ist gemäss Zürcher Lehrpersonalgesetz verpflichtet, den Unterricht gewissenhaft vorzubereiten und $\mathrm{zu}$ gestalten. 47 Sie hat die obligatorischen Lehrmittel und Lernmaterialien $\mathrm{zu}$ verwenden. ${ }^{48}$ Im Übrigen gilt die Methodenfreiheit.49 Lehrpersonen haben damit vergleichsweise grosse Spielräume, die konkrete Unterrichtstätigkeit nach ihren Vorstellungen zu gestalten. Damit verbinden sich gerade in politischer Hinsicht erhebliche Möglichkeiten der Beeinflussung, da sie meist Kinder oder Jugendliche unterrichten, die sich in vielen Fragen noch kein selbstständiges Urteil bilden können. 50

21 Das Gebot der politischen Neutralität soll in dieser Hinsicht Grenzen setzen. Es ist von grosser praktischer Bedeutung und tagtäglich in unzähligen Schulstunden direkt relevant. Wissenschaftliche Interpretationen dazu sind allerdings eher rar. Eine Auseinandersetzung mit der Thematik findet sich bei einzelnen Autoren, deren Stellungnahmen im Folgenden näher zu betrachten sind:

22 Peter Hänni analysiert die Thematik unter dem Aspekt der Treuepflicht, behandelt die Rolle der Lehrperson in diesem Zusammenhang aber auch grundsätzlich.

$4 7 \longdiv { \S 1 8 \text { Abs. } 2 \text { Satz } 1 \text { LPG. Vgl. Tobias Jaag/Markus } }$ Rüssli, Staats- und Verwaltungsrecht des Kantons Zürich, 5. Aufl., Zürich 2019, S. 383.

$48 \S 18$ Abs. 2 Satz 2 LPG. Vgl. Tobias Jaag, Rechtsfragen der Volksschule, insbesondere im Kanton Zürich, in: Schweizerisches Zentralblatt für Staats- und Verwaltungsrecht 98 (1997), 537562, 551; Plotke (Fn. 27), S. 570.

$49 \S 18$ Abs. 2 Satz 3 LPG. Vgl. Plotke (Fn. 27), S. $566 \mathrm{ff}$.

5o Plotke (Fn. 27), S. 573. 
In Anlehnung an den Philosophen Hans Saner vertritt er die Auffassung, dass die Vorstellung einer unpolitischen oder neutralen Lehrperson reine Fiktion sei. Eine Lehrerin oder ein Lehrer sei als Pädagogin oder Pädagoge unvermeidlich in einer politischen Rolle.51 Diese Rolle werde besonders dort greifbar, wo politische Fragen und Probleme zum Inhalt des Unterrichts werden. Die Lehrperson solle beispielsweise aufzeigen, wie und wodurch politische Freiheit und Gerechtigkeit ermöglicht oder unterdrückt werden. «Dabei ist das Engagement des Lehrers in der politischen Bildung nicht zu verwechseln mit Indoktrination und politischer Propaganda, denn es geht ja nicht darum, den Schüler für eine bestimmte politische Meinung zu gewinnen, sondern um die Herausbildung der politischen Urteilsfähigkeit.»52 Die Lehrperson dürfe ihre Meinung bekennen, wenn sie diese rational begründe und wenn sie sich auch zum Anwalt von Alternativen mache. 53

Herbert Plotke vertritt die Ansicht, dass der Unterricht «Kenntnisse und Informationen religiösen und weltanschaulichen Charakters vermitteln [darf], solange er objektiv, ohne Indoktrination oder Wertung erteilt wird und auf die verschiedenen Standpunkte eingeht.»54 Die Lehrperson dürfe ihre Meinung darlegen, aber nicht aufdrängen, müsse also andere Ansichten gelten lassen. Traue sich eine Lehrperson die nötige Sachkenntnis und Objektivität nicht zu, so solle sie Schülerinnen und Schüler die einzelnen Auffassungen in Vorträgen darstellen lassen oder bei aktuellen und besonders umstrittenen Fragen Referenten, Kollegen oder andere Personen beiziehen. 55

24 Nach Markus Rüssli bedeutet politische Neutralität nicht, dass politisch kontroverse Themen oder religiöse Fragen vom Unterricht auszuklammern wären. Die Lehrkräfte müssten aber für eine ausgewogene Darstellung bzw. Diskussion sorgen. «Sie selbst haben unparteiisch zu bleiben und dürfen nicht in einseitiger Weise Einfluss auf die Schülerinnen und Schüler nehmen. Politische Indoktrination, [...] das Schlechtmachen einzelner Glaubensbekenntnisse oder Weltanschauungen oder das Propagieren von Ideen, die sich mit den Grundwerten des demokratischen Staatswesens nicht vereinbaren lassen, ist den Lehrpersonen untersagt.» 56

25 In diesen Äusserungen werden unterschiedliche Aspekte sichtbar. In die Richtung einer strengen Neutralitätspflicht weisen Forderungen, dass der Unterricht «ohne Wertung» 57 erfolgen solle und die Lehrkräfte «unparteiisch» 58 zu bleiben hätten. In die Richtung grösserer Spielräume gehen dagegen Aussagen wie die, dass eine Lehrperson ihre Meinung bekennen dürfe, solange sie diese rational begründe und andere Ansichten gelten lasse. 59

\section{Eigener Vorschlag: Kriterien}

26 Aufbauend auf den referierten Deutungen soll im Folgenden ein eigener Vorschlag entwickelt werden, das Gebot der

55 Plotke (Fn. 27), S. 39.

${ }_{56}$ Rüssli (Fn. 3), N. 14.

57 Oben bei Fn. 54 .

5 Oben bei Fn. 56 .

59 Oben bei Fn. 53, 55 . 
politischen Neutralität von Lehrpersonen zu interpretieren. Ausgangspunkt sollen die Schülerinnen und Schüler und deren Grundrechte bilden. Die politische Neutralität der Schule ist kein Selbstzweck, sondern hat den Sinn, eine freie Meinungsbildung und Willensbildung der Schülerinnen und Schüler zu ermöglichen. Analog dazu liegt auch das Gebot der konfessionellen beziehungsweise religiösen Neutralität des Unterrichts darin begründet, dass die Schülerinnen und Schüler in ihrer religiösen Freiheit nicht einschränkt werden sollen.60 Aus dieser Warte ist klar, dass jede Form systematischer Beeinflussung (Indoktrination) der politischen Haltung der Schülerinnen und Schüler unzulässig wäre. Schwieriger ist die Frage, ob und gegebenenfalls in welcher Form eine Lehrkraft ihre eigene politische Meinung im Unterricht äussern darf.

Geht man von den rechtlich geschützten Interessen der Schülerinnen und Schüler aus, so ist die blosse Aussage einer Lehrperson, wo sie in einer Frage politisch steht, nicht unter allen Umständen als Pflichtverstoss zu werten. Eine Äusserung dieser Art muss die politische Urteilsbildung von Schülerinnen und Schülern nicht unbedingt beeinträchtigen, ja sie kann sie unter bestimmten Bedingungen sogar fördern. Mitunter ist eine solche Positionierung der Lehrperson sogar gewünscht, um in der Auseinandersetzung mit deren Haltung besser ein eigenes Urteil bilden zu können. ${ }^{61} \mathrm{Zu}$ beach-

6o Vgl. Lorenz Engi, Die religiöse und ethische Neutralität des Staates. Theoretischer Hintergrund, dogmatischer Gehalt und praktische Bedeutung eines Grundsatzes des schweizerischen Staatsrechts, Habil. St. Gallen, Zürich 2017, § 9.

61 Vgl. - allerdings bezüglich des Unterrichts auf Hochschulstufe - die Aussage Alois Riklins: ten ist in diesem Zusammenhang auch, dass es Lehrerinnen und Lehrer gibt, die sich beispielsweise in einer politischen Partei engagieren, so dass ihr politischer Standort den Schülerinnen und Schülern und deren Eltern ohnehin bekannt ist. Auch ohne formelle Parteimitgliedschaft wird die politische Positionierung einer Person zudem an vielen kleinen Dingen ohnehin häufig erkennbar (fährt der Lehrer mit dem Auto oder dem Velo zur Schule etc.).

28 Eine einfache bekennende Aussage zur politischen Position - sei es in einer konkreten politischen Streitfrage oder in allgemeiner Weise - kann daher mit der politischen Neutralität vereinbar sein, soweit einige wesentliche Bedingungen eingehalten werden. $\mathrm{Zu}$ verlangen ist in diesem Fall

1. dass die Lehrperson die Meinung klar als persönliche Auffassung deklariert,

2. dass sie ihre Meinung lediglich sachlich darlegt, aber nicht für sie wirbt, das heisst die Schülerinnen und Schüler nicht davon zu überzeugen versucht, und

3. dass sie im gleichen Umfang auf andere politische Ansichten eingeht. Die Vor- und Nachteile der relevanten politischen Positionen müssen gleichberechtigt thematisiert werden. So wäre es etwa nicht zulässig, bei der Diskussion einer Abstimmungs-

\footnotetext{
«[M]eine Studenten gaben sich nicht damit zufrieden, einen politischen Eunuchen vor sich zu haben, der für praktisch-politische Stellungnahmen nur ausserhalb des Hörsaals zur Verfügung stehen wollte.» (Geleitwort, in: Engagierte Politikwissenschaft - Ausgewählte Schriften, Bern 2018, S. 7-34, 9 f.).
} 
frage der einen Seite viel mehr Zeit einzuräumen als der anderen.

29

Zudem ist selbstverständlich vorauszusetzen, dass die Lehrperson andere Meinungen, die von Schülerinnen und Schülern vorgebracht werden, vorbehaltlos akzeptiert und deren Äusserung in keiner Weise mit Nachteilen für die Betroffenen verknüpft.

30 Diese allgemeinen Voraussetzungen sind nach Schulstufe bzw. Alter der Schülerinnen und Schüler zu differenzieren. Ältere Schüler sind in der Regel gut in der Lage, eine als solche deklarierte persönliche Meinung einer Lehrperson einzuordnen. Bei jüngeren Schülern ist mehr Zurückhaltung geboten.

Werden diese Bedingungen eingehalten, erscheint es möglich, die freie politische Willensbildung und Entwicklung von Schülerinnen und Schülern zu gewährleisten, auch wenn eine Lehrperson ihren politischen Standpunkt deklariert. Eine krampfhafte Verschleierung desselben kann unter Umständen kontraproduktiv sein, da die Lehrerin oder der Lehrer die eigenen Präferenzen unter Umständen doch in irgendeiner Weise einfliessen lässt und die Schülerinnen und Schüler so subtil beeinflusst. Legt die Lehrperson dagegen ihre Haltung offen, können sich die Schülerinnen und Schüler damit auseinandersetzen, was ihrer politischen Entwicklung nicht hinderlich $\mathrm{zu}$ sein braucht. ${ }^{62}$

62 In diesem Sinn auch der Zürcher Stadtrat in einer Interpellationsantwort: «Die Verpflichtung $\mathrm{zu}$ den Grundwerten des demokratischen Staatswesens sowie zu den demokratischen Wertvorstellungen schliesst ein, dass in der Volksschule durchaus politische Meinungen Platz haben dürfen. Sie müssen jedoch ausgewogen dargestellt
Hierbei kann ein Vergleich zum Thema der Lehrerin mit Kopftuch gezogen werden: Das Bundesgericht hat 1997 ein Verbot im Kanton Genf, mit Kopftuch zu unterrichten, geschützt. Indes bestanden im Kanton Genf dafür besondere rechtliche Grundlagen. Ohne solche Grundlagen wäre ein pauschales Kopftuchverbot für Lehrerinnen nach wohl überwiegender Lehrmeinung nicht zulässig. 63 Trägt eine Lehrerin ein Kopftuch, so deklariert sie ebenfalls ihre persönliche Meinung, in diesem Fall in religiösen Belangen. Dies soll sie, folgt man zahlreichen Lehrmeinungen, tun dürfen, jedoch ist es ihr nicht erlaubt, für diesen Glauben in irgendeiner Weise zu werben. Ähnlich kann eine Lehrperson ihre persönliche politische Meinung deklarieren, ohne jedoch im Rahmen des schulischen Unterrichts für diese werben zu dürfen.

\section{Lehrmittel}

32 Neben der Unterrichtstätigkeit sind hinsichtlich der politischen Neutralität vor allem die Lehrmittel von Interesse. Als Lehrmittel gelten alle Unterrichtsmittel, insbesondere Bücher, Software, Filmund Audiomaterial.64 Der Bildungsrat kann im Kanton Zürich bestimmte

bzw. unterschiedliche Standpunkte müssen gleichwertig behandelt werden.» (Interpellation von Yasmine Bourgeois, Christian Huser und zehn Mitunterzeichnenden betreffend Lehrmittel und unterrichtsergänzende Angebote der Stadt an den Volks-, Berufs- und Kantonsschulen sowie Instrumente zur Sicherstellung der politischen und konfessionellen Neutralität, GR Nr. 2018/330, Antwort des Stadtrates vom 27. Februar 2019).

63 Vgl. Lorenz Engi, Das Kopftuch in öffentlichen Institutionen - Aktuelle Fragen insbesondere im Zusammenhang mit der Zulassung von Pädagogik-Studentinnen zu Praktika; in: Aktuelle Juristische Praxis 2019, S. 208-217, 212 ff.; ders. (Fn. 60), S. $420 \mathrm{ff}$.

$64 \S 19$ Abs. 1 Volksschulverordnung vom 28. Juni 2006 (VSV; LS 412.101). 
Lehrmittel für obligatorisch erklären. ${ }^{65}$ Diese Lehrmittel sind im Unterricht zu verwenden. ${ }^{66}$ Die obligatorischen Lehrmittel bilden eine Schranke der Lehrund Methodenfreiheit. § 23 VSG bestimmt in diesem Sinn: «Die Lehrperson hat das Recht, im Rahmen des Lehrplans, der obligatorischen Lehrmittel, des Schulprogramms und der Beschlüsse der Schulkonferenz den Unterricht frei zu gestalten.»

Die Lehrpersonen haben bei der Wahl der Lehrmittel ein Mitwirkungsrecht. § 4 der Zürcher Lehrmittelverordnung legt fest, dass die Bildungsdirektion die Mitwirkung der Lehrpersonen der Volksschule bei der Schaffung und Beschaffung von Lehrmitteln sicherstellt und die Lehrpersonen über die Planung im Bereich der obligatorischen Lehrmittel informiert. Im Weiteren hat die Lehrmittelkommission, die zu einem massgeblichen Teil aus Lehrpersonen besteht, 67 in diesem Bereich Kompetenzen. Gemäss $\S 6$ der Lehrmittelverordnung berät sie den Bildungsrat, das Volksschulamt und den Lehrmittelverlag bei der Ausrichtung des kantonalen Lehrmittelwesens, der Planung im Bereich der obligatorischen Lehrmittel und in anderen Fragen.

34 Im Bereich der Lehrmittel folgen aus dem Gebot der politischen Neutralität der öffentlichen Schule höhere Anforderungen als bei der Unterrichtstätigkeit von Lehrpersonen. Denn während die

$6 5 \longdiv { \S 2 2 \text { Abs. } 1 \text { Volksschulgesetz vom 7. Februar 2005 } }$ (VSG; LS 412.100). Vgl. § 2 Lehrmittelverordnung für die Volksschule vom 20. August 2014 (LS 412.14). Das Volksschulamt des Kantons Zürich hat die Fachbereiche mit LehrmittelObligatorium publiziert.

$66 \S 19$ Abs. 2 VSV.

67 Vgl. § 5 Lehrmittelverordnung (Fn. 65).
Stellungnahme einer Lehrperson als persönliche Meinung klar wahrnehmbar ist, hat das Lehrmittel einen unpersönlicheren, objektiveren Charakter. Politische Meinungen, die darin enthalten sind, könnten schwerer als persönliche Auffassung eingeordnet und insoweit relativiert werden. Es ist auch kaum möglich, allfällige Einseitigkeiten in einem Lehrmittel konsequent und befriedigend in jedem Fall auszugleichen. So beschäftigen sich die Schülerinnen und Schüler auch ausserhalb der Schule stark mit ihren Lehrmitteln, beispielsweise bei Hausaufgaben oder Prüfungsvorbereitungen. Dabei sind keine Korrekturen durch Lehrpersonen möglich. Auch aus zeitlichen und anderen Gründen ist es kaum möglich, einseitige politische Darstellungen in einem Lehrbuch konsequent und ausreichend zu korrigieren.

35 Bezüglich politischer Aussagen in Lehrmitteln ist daher grosse Zurückhaltung geboten. Bezüglich politisch umstrittener Fragen ist eine einseitige Positionierung eines Lehrmittels unzulässig. Soweit allgemeine gesellschaftspolitische Aussagen notwendig erscheinen - etwa bezüglich des friedlichen und toleranten Zusammenlebens von Religionsgemeinschaften -, müssen sie den gesellschaftlichen Grundwerten entsprechen, die in der Verfassung zum Ausdruck kommen. Parteiische Standpunkte können in einem Lehrmittel nur wiedergegeben werden, wenn sie als solche klar deklariert und alle anderen relevanten Auffassungen ebenfalls dargestellt werden.

36 Jüngst gab es im Kanton Zürich zur Frage politisch neutraler Lehrmittel mehrere politische Vorstösse. Auslöser war die Diskussion um das Lehrmittel «Gesell- 
schaften im Wandel», das in der medialen Berichterstattung als politisch einseitig dargestellt wurde. ${ }^{68} \mathrm{Im}$ Zürcher Kantonsrat wurden zwei parlamentarische Initiativen, eine Interpellation sowie eine dringliche Anfrage eingereicht. ${ }^{69}$ Die beiden parlamentarischen Initiativen zielen darauf $a b$, die Zuständigkeiten für die Sicherstellung der politischen Neutralität klarer zu regeln sowie die Neutralität der Lehrmittel rechtlich explizit zu verankern. Der Regierungsrat hielt in seinen Antworten auf die Vorstösse bezüglich der Lehrmittel unter anderem fest: «Der politischen Neutralität von Lehrmitteln, die im Volksschulunterricht eingesetzt werden, kommt eine grosse Bedeutung zu. [...] Ein politisch ausgewogener Unterricht vermittelt die verschiedenen Standpunkte und Positionen innerhalb der Gesellschaft. [...] Kein Lehrmittel kann zu jedem Thema alle unterschiedlichen Haltungen und Meinungen darlegen. Der Anspruch auf Ausgewogenheit ist jedoch über das gesamte Lehrmittel hinweg zu gewährleisten, was auch für

68

Lektion 1 - ein Loblied auf die Unia. In neuen Lehrmitteln wird politische Werbung verbreitet, NZZ vom 31. August 2018, S. 13.

69 Parlamentarische Initiative von Marc Bourgeois (FDP, Zürich), Bettina Balmer (FDP, Zürich) und Anita Borer (SVP, Uster) vom 17. September 2018 betreffend «Politisch und konfessionell neutrales öffentliches Bildungswesen, neutrale Lehrmittel und ausgewogene unterrichtsergänzende Angebote», KR-Nr. 288/2018; Parlamentarische Initiative von Bettina Balmer (FDP, Zürich), Marc Bourgeois (FDP, Zürich) und Anita Borer (SVP, Uster) vom 17. September 2018 betreffend «Politische Neutralität bei Lehrmitteln für die Volksschule», KR-Nr. 287/2018; Dringliche Anfrage «Politisch neutrale Lehrmittel sicherstellen», KRNr. 291/2018, vom Regierungsrat beantwortet mit Regierungsratsbeschluss (RRB) 954/2018 vom 3. Oktober 2018; Interpellation «Politische Neutralität der Volksschule», KR-Nr. 290/2018, vom Regierungsrat beantwortet mit RRB 955/2018 vom 3. Oktober 2018. das genannte Lehrmittel gilt.»70

\section{Grundsätzlich: Neutralität als Grenze}

37 Im Licht der bisherigen Ergebnisse kann die eingangs erwähnte, grundsätzliche Frage wieder aufgegriffen werden, ob und inwiefern sich staatliche Angestellte in ihrer amtlichen Tätigkeit von persönlichen Überzeugungen distanzieren können und müssen. Teilweise wird die Möglichkeit einer solchen Distanzierung bezweifelt.71 Die Frage kann im vorliegenden Zusammenhang nicht in ihrer ganzen Tiefe behandelt werden. Einige Schlussfolgerungen bezüglich des Spannungsfeldes zwischen staatlicher Neutralität und persönlicher Überzeugung und hinsichtlich eines realistischen Neutralitätsverständnisses lassen sich jedoch ziehen.

$38 \mathrm{Zu}$ berücksichtigen sind verschiedene Gesichtspunkte. Auf der einen Seite ist es ein Aspekt der Professionalität, in einer beruflichen Rolle individuelle politische oder religiöse Überzeugungen insoweit zurückzustellen, als hoheitliches Handeln in Frage steht. Dies erscheint grundsätz-

70 RRB 954/2018, Antwort zu Frage 1. Zum Verhalten der Lehrpersonen vgl. insb. RRB 955/2018, Antwort zu Frage 7. Auch in anderen Kantonen gab es entsprechende Vorstösse, z.B. im Kanton Solothurn: Auftrag Fraktion FDP. Die Liberalen: Konfessionell und politisch neutrale Lehrmittel, KR-Nr. A 0119/2018 (DBK), dazu RRB 2018/1815 vom 20. November 2018. Der Auftrag wurde von der Bildungs- und Kulturkommission am 18. Dezember 2018 einstimmig für erheblich erklärt.

${ }_{71}$ Vgl. Markus Müller, Religion im Rechtsstaat Von der Neutralität zur Toleranz, Bern 2017, insb. S. 84 ff. Zum Hintergrund (Unmöglichkeit innerer Unabhängigkeit gegenüber persönlichkeitskonstitutiver Vorbestimmtheit) auch ders., Psychologie im öffentlichen Verfahren - Eine Annäherung, Bern 2010, insb. S. 73 ff. 
lich möglich. Die Unterscheidung von amtlicher Tätigkeit, die sich an Rechtsnormen ausrichtet, und persönlichen Überzeugungen ist täglich vielfach praktizierte Realität. So wird etwa ein Staatsangestellter, der Wahl- und Abstimmungsnormen $\mathrm{zu}$ vollziehen hat und gleichzeitig Mitglied einer Partei ist, diese Partei nicht bevorzugen, sondern gleich behandeln wie andere Parteien in der gleichen Situation. Fraglos gibt es für dieses Zurückstellen individueller Präferenzen Grenzen. Zu denken ist etwa an Ermessensspielräume, die in einer Weise genutzt werden können, die dem handelnden Akteur entspricht. Auch der (im demokratischen Rechtsstaat allerdings sehr seltene) Fall, dass Normen tiefen moralischen Überzeugungen der oder des Betreffenden widersprechen, ist zu bedenken ${ }^{72}$.

39 Die Leitidee, dass Normen auch dann vollzogen werden, wenn sie den individuellen Präferenzen der handelnden Personen nicht entsprechen, ist für den Rechtsstaat fundamental. Die Bürgerin oder der Bürger muss sich darauf verlassen können, im Kontakt mit staatlichen Institutionen nach allgemeinen Massstäben, nicht nach den individuellen Überzeugungen des jeweiligen Akteurs behandelt zu werden. Würden wir an den realistischen Gehalt dieser Idee nicht glauben, wäre die Rechtsordnung in letzter Konsequenz überflüssig beziehungsweise nur (un)schöner Schein. Jeder Mensch ist denn auch hochgradig geübt darin, mit verschiedenen Rollen umzugehen und sein Verhalten je nach Kontext im Sinne jeweiliger Adäquanz zu

$72 \overline{\text { Vgl. zu dieser Problematik Anne Kühler, Das }}$ Grundrecht der Gewissensfreiheit, Diss., Bern 2012, S. $279 \mathrm{ff}$. variieren.73 Bei Lehrerinnen und Lehrern im Besonderen gibt es ein rollenadäquates Verhalten, das sie beherrschen müssen und welches die betreffende Schulleitung durchzusetzen hat. Im Zusammenhang mit der politischen Neutralität der Schule heisst dies unter anderem, dass die Lehrperson im schulischen Rahmen nicht aktiv für bestimmte - persönlich für wichtig gehaltene - politische Positionen werben darf. Sie würde ihre berufliche Stellung durch ein solches Verhalten missbrauchen.

40 Auf der anderen Seite ist jedoch auch zu berücksichtigen, dass kein Mensch sich vollständig «neutralisieren» kann (und soll). Die persönlichen Merkmale und Überzeugungen (politischer, religiöser oder anderer Art) prägen das Verhalten, oft unbewusst. Bis in den Habitus, die Kleidung, den Sprachgebrauch und andere Dinge hinein werden persönliche Werthaltungen oft erkennbar und wirksam. Gerade die Aufgabe einer Lehrerin oder eines Lehrers geht mit so vielen Handlungsspielräumen einher, dass eine persönliche Färbung des Handelns unausweichlich ist. Aus diesen Gründen ginge die Erwartung fehl, dass eine Lehrperson (und eine staatliche Angestellte oder ein staatlicher Angestellter generell) in keiner Weise als politisch irgendwie gebunden erscheinen darf. Dies liefe auf das falsche Ideal hinaus, dass eine Lehrperson quasi als roboterhafter Automat zu funktionieren hätte - etwas, was sie als gute Pädagogin beziehungsweise guter Pädagoge gerade nicht sein soll. Eine Lehrerin oder ein Lehrer sollte persön-

$73 \overline{\text { Wer beispielsweise nicht versteht, dass er gegen- }}$ über seinen Mitarbeitern anders kommunizieren sollte als gegenüber seinen Kindern, hat früher oder später ein Problem. 
lich fassbar sein, und dies kann bei der Diskussion aktueller gesellschaftlicher Fragen durchaus bedeuten, dass er oder sie mit einer eigenen Meinung erkennbar wird.

41 Diese vielfältigen Aspekte lassen sich so verbinden, dass die (politische) Neutralität eher als Grenze denn im Sinne durchgängiger inhaltlicher Determinierung zu verstehen ist. Die Neutralität meint nach diesem Verständnis nicht, dass eine Lehrperson in keiner Weise mit ihren politischen Präferenzen erkennbar sein soll - dies wäre unrealistisch und wahrscheinlich auch nicht sachgerecht. Die Neutralitätsforderung ist vielmehr so zu verstehen, dass in Bezug auf gewisse, allzu weitgehende Manifestationen persönlicher Haltungen Grenzlinien gezogen werden. Hinter einem tauglichen Neutralitätskonzept sollte mit anderen Worten nicht eine perfektionistische Vision absoluter Neutralität stehen, sondern eher die Idee eines notwendigen Korrektivs allzu weitgehender Einseitigkeiten.74 So verstanden, lässt die Neutralitätsforderung erheblichen Spielraum.75 Diesseits der Grenze, die sie setzt, im Bereich des Erlaubten, stehen viele Möglichkeiten adäquaten Handelns offen. So dürfte es, wie erwähnt, zulässig sein, wenn eine Lehrperson eine politische Meinung äussert, sofern andere Auffassungen gleichberechtigt zum Tragen kommen und die Schülerinnen und Schüler sich frei ein eigenes Urteil bilden können.

\section{Schluss}

42 Zahlreiche kantonale Normen schreiben vor, dass der schulische Unterricht politisch neutral zu sein habe. Der Sinn solcher Bestimmungen liegt vor allem darin, die freie Willensbildung der Schülerinnen und Schüler zu gewährleisten. Dies macht alle Bestrebungen unzulässig, die Schülerinnen und Schüler in einer bestimmten Weise politisch lenken zu wollen. Gleichzeitig kann eine Lehrperson sich selbst nicht vollständig neutralisieren. Eine absolute Neutralität im Sinne gänzlicher Nichterkennbarkeit politischer Präferenzen dürfte eine Illusion sein. Wichtig ist es daher, dass eine Lehrperson, die eine politische Meinung äussert, diese klar als solche deklariert und anderen Positionen und Argumenten gleichen Raum gibt.

43 So betrachtet, bildet die Neutralitätsforderung eine Art Leitplanke. Sie legt das Verhalten nicht in allen Details fest, markiert aber Grenzen. Das (politische) Neutralitätsgebot lässt weite Spielräume. Diese situationsgerecht zu nutzen, ist die Pflicht einer Lehrperson. Abstrakte Normen können eine gute Unterrichtspraxis nur bedingt vorwegnehmen. Striktere Massstäbe gelten bezüglich der Lehrmittel. Diese dürfen, soweit politisch kontroverse Fragen berührt sind, keine einseitige Färbung aufweisen, sondern müssen insgesamt ausgewogen sein.
74 Vgl. auch M. Müller, Psychologie (Fn. 71), S. 93 ff.

75 Vgl. im Kontext der religiösen Neutralität Bernhard Ehrenzeller, Glauben, Gewissen und Weltanschauung, in: Detlef Merten/Hans-Jürgen Papier (Hrsg.), Handbuch der Grundrechte in Deutschland und Europa, Band VII/2, Heidelberg/Zürich/St. Gallen 2007, S. 301-325, Rz. 31. 